\title{
Authors reply to Dr. Kawada's comments
}

\author{
Tsan Yang $\cdot$ Chien-An Sun
}

Received: 26 April 2013/Accepted: 1 August 2013/Published online: 13 August 2013

(C) Springer Science+Business Media New York 2013

We thank Dr. Kawada for his insightful comments [1] on our study [2], which highlights the importance of the C-reactive protein (CRP) concentration as a significant risk marker for metabolic syndrome (MetS). Dr. Kawada argued that stratified analyses by gender for the association between CRP concentrations and MetS should be performed in our study, given that there were gender differences in the odds ratios of CRP concentrations for MetS. Our study demonstrates that a significant stepwise increase in the prevalence of MetS across quartiles of CRP was observed in both men and women. Therefore, the CRP concentration is a significant risk marker for MetS in Chinese adults. However, previous studies have suggested that chronic inflammation, as indicated by elevated CRP concentration, might have a greater effect on the development of MetS in women than in men [3, 4]. Ethnic differences in CRP levels and the prominent individual components of MetS may account for the inconsistent observations for gender-specific associations between CRP and MetS among various ethnic groups.

Dr. Kawada noted a sizable difference in MetS prevalence between Mahajan et al. [5] study in India and our study in Taiwan. It has been noted that the prevalence of MetS has reached epidemic proportion in India [6]. Thus, our study might include relatively more sub-clinical MetS subjects. We agree with Dr. Kawada's point that risk

\section{T. Yang}

Department of Health Business Administration, Meiho

University, Neipu, Pingtung County, Taiwan

C.-A. Sun $(\bowtie)$

Department of Public Health, College of Medicine, Fu-Jen

Catholic University, New Taipei, Taiwan

e-mail:040866@mail.fju.edu.tw assessment should be conducted by understanding the baseline characteristics of the target population.

Dr. Kawada emphasized the need for evaluating the subclinical association between CRP and MetS components. In particular, elevated CRP was significantly associated with the risk of impaired fasting glucose and/or impaired glucose tolerance [5]. Therefore, CRP might interplay with insulin resistance and have central role in the pathophysiology of MetS.

Conflict of interest The authors declare no conflict of interest.

\section{References}

1. T. Kawada, Serum C-reactive protein and metabolic components. Endocrine. (2013). doi:10.1007/s12020-013-9966-2

2. T. Yang, C.H. Chu, P.C. Hsieh, C.H. Hsu, Y.C. Chou, S.H. Yang, C.H. Bai, S.L. You, L.C. Hwang, T.C. Chung, C.A. Sun, C-reactive protein concentration as a significant correlate for metabolic syndrome: a Chinese population-based study. Endocrine 43, 351-359 (2013)

3. M.K. Rutter, J.B. Meigs, L.M. Sullivan, R.B. D'Agostino Sr, P.W. Wilson, C-reactive protein, the metabolic syndrome, and prediction of cardiovascular events in the Framingham Offspring Study. Circulation 110, 380-385 (2004)

4. N. Nakanishi, T. Shiraishi, M. Wada, C-reactive protein concentration is more strongly related to metabolic syndrome in women than in men. Circ. J. 69, 386-391 (2005)

5. A. Mahajan, A. Jaiswal, R. Tabassum, A. Podder, S. Ghost, S.V. Madhu, S.K. Mathur, N. Tandon, D. Bharadwaj, Elevated levels of C-reactive protein as a risk factor for metabolic syndrome in Indians. Atherosclerosis 220, 275-281 (2012)

6. A. Ramachandran, S. Mary, A. Yamuna, N. Murugesan, C. Snehalatha, High prevalence of diabetes and cardiovascular risk factors associated with urbanization in India. Diabetes Care 31, 893-898 (2008) 